\title{
The prevalence of seizures in children with developmental delay
}

Raidah Albaradie, MD, Hafiz Habibullah, MD, Ali Mir, MD, Alaa K. Alshammari, MBBS, Modhi S. Alajmi, MBBS, Fatima A. Alsubaie, MBBS, Reem R. Alsudairi, MBBS, Shahid Bashir, PhD.

\section{ABSTRACT}

الأهداف : دراسة مدى انتشار التشنجات في الاطفال المصابين بالتأخر

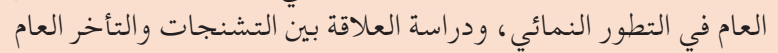

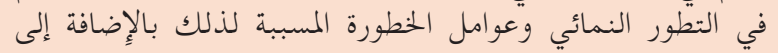

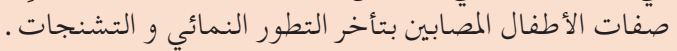

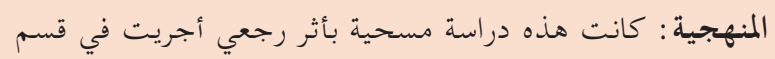

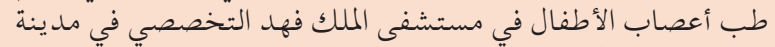

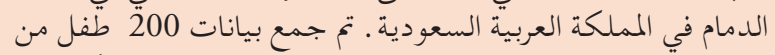

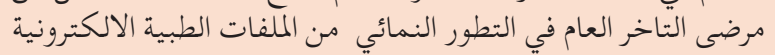

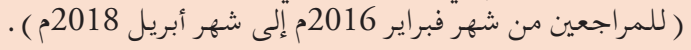

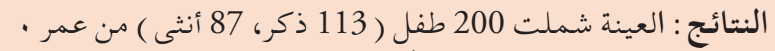

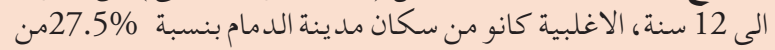

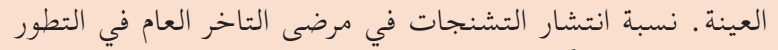

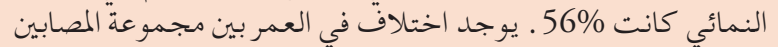

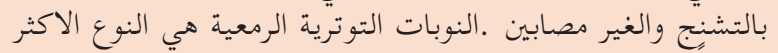

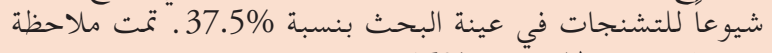

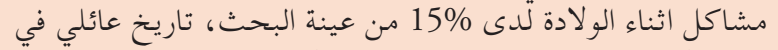

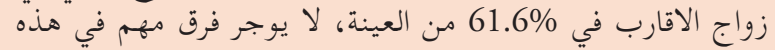

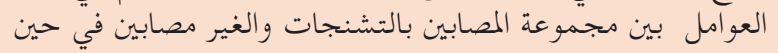

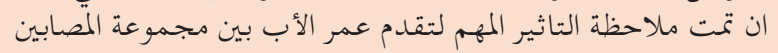

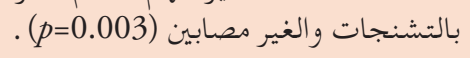

الخلاصة : نسبة انتشار التشنجات كانت عالية لدى الأسف الطفال المصابين

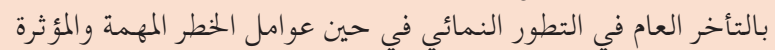

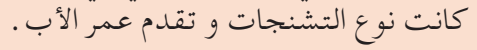

Objectives: To study the prevalence of seizures in children with GDD and identify the characteristics of such patients; to examine the association of GDD with epilepsy and to determine the effect of certain risk factors on this association.

Methods: A retrospective cross-sectional study conducted at the pediatric neurology and developmental assessment clinic at King Fahad specialist hospital (KFSH), Saudi Arabia. All data were collected by reviewing the electronic medical records of 200 pediatric patients who presented with global developmental delay from February 2016 to April 2018.

Results: The sample includes 200 children (113 males, 87 females) aged zero to 12 years. The largest group of participants came from the Dammam region, representing $27.5 \%$ of the sample. The prevalence of epilepsy in GDD patients was 56\%; the epilepsy and non-epilepsy groups differed significantly in age. The most common type of seizure was generalized onset motor, which were observed in $37.5 \%$ of the sample. Problems during labor occurred in $15 \%$ of the sample; consanguineous marriage occurred in $61.6 \%$ of the participants. Neither of these factors differed significantly in the epilepsy and non-epilepsy groups. Advanced paternal age did differ significantly in the two groups $(p=0.003)$.

Conclusion: The prevalence of epilepsy is high in children with GDD, and of the factors studied here, the most significant variables affecting this correlation are the type of seizure and advanced paternal age.

Neurosciences 2021; Vol. 26 (2): 186-191 doi: 10.17712/nsj.2021.2.20200106

From the Department of Pediatric neurology (Albaradie, Habibullah, Mir), Neuroscience Center (Bashir), King Fahad Specialist Hospital, Dammam, from the College of Medicine (Alshammari, Alajmi, Alsubaie, Alsudairi), Imam Abdulrahman Bin Faisal University, Dammam, Kingdom of Saudi Arabia.

Received 13th August 2020. Accepted 8th January 2021.

Address correspondence and reprint request to: Dr. Raidah S. AlBaradie, Neuroscience Center, King Fahad Specialist Hospital, Dammam, Kingdom of Saudi Arabia. E-mail: Raidah.albaradie@ kfsh.med.sa

ORCID ID: https://orcid.org/ 0000-0001-7575-7671

Trus lobal developmental delay (GDD) is a general term I used when children under the age of five years' experience significant delays in 2 or more developmental domains (daily living activities, motor skills, speech/ language, cognitive abilities, and social/emotional skills). The precise prevalence of GDD is unknown, but it is estimated to be $1 \%-3 \% .{ }^{1}$ Several studies have proposed 
an association between GDD and epilepsy. ${ }^{1-3}$ Seizures are common; $4-10 \%$ of children experience at least one episode of seizures. Abnormal excessive or synchronous neuronal activity in the brain cause seizure and result in transient signs and/or symptoms. ${ }^{4}$ According to the International League Against Epilepsy (ILAE) patient can be diagnosed with epilepsy if the probability of further seizures after first unprovoked seizure similar to the general recurrence risk of at least $60 \%$ after 2 unprovoked seizures, occurring over the next 10 years. ${ }^{5}$ About $30 \%$ of children with epilepsy have behavioral or cognitive impairments. ${ }^{6}$ Epilepsy has been associated with neurocognitive impairment in children, and it can adversely impact school performance and long-term psychosocial prospects. ${ }^{7,8}$ The impact of epilepsy on GDD can be explained by the effect of seizures on developing brain structures and functions and on repeated brain volume reduction (especially white matter tissue, which is seen in patients with childhoodonset temporal lobe epilepsy). Brain reduction is also associated with poor cognitive skills. ${ }^{9,10,11}$ However, cognitive impairments in general often go undiagnosed; therefore, children with epilepsy should be periodically screened for GDD to enable early intervention and to maximize these patients' chances for academic, professional, and social success. , $3,6,7,9^{2}$

Little research has been carried out aiming to address the etiological factors of GDD in children attending a tertiary care hospital. This study was the only one study was done in Saudi Arabia in 2019 that focused on the prevalence of epilepsy in pediatric patients with developmental delay in Saudi Arabia, as it found that $56 \%$ of Saudi children with GDD have epilepsy. The same study showed that multiple variables were found to be related to GDD including consanguineous marriage (57\%), and low birth weight (22\%). Causes identified includes neuroradiology abnormalities in 58\% of GDD children, 45\% had abnormal EEG, genetic defects in $40 \%$, and lastly metabolic disorders in one quarter of the participants. ${ }^{1}$

The aim of the present study is to examine the prevalence of epilepsy in children who visited a development clinic and the demographic characteristics of these patients. It will also examine the effects of other factors such as type of seizures, problems during

Disclosure. Authors have no conflict of interests and the work was not supported or funded by any drug company. pregnancy or labor, and advanced maternal or paternal age.

Methods. Study subjects. This is a retrospective cross-sectional study conducted at the pediatric neurology and developmental assessment clinic at King Fahad specialist hospital (KFSH), Kingdom of Saudi Arabia. This study included 200 pediatric patients aged zero to 12 years who visited the clinic from February 1, 2016 to April 1, 2018. Participants were selected using convenience sampling. All included children were referred to the pediatric neurology clinic and underwent multidisciplinary developmental assessment involving a detailed history and a developmental pediatric examination. Inclusion criteria were (1) children with zero to 12 years of both genders, (2) children diagnosed with epilepsy were confirmed by pediatric epileptologist (RB and AM). Exclusion criteria were (1) children with missing information, (2) children diagnosed with autism, cerebral palsy, CNS infections and (3) children who did not complete the developmental assessment.

All data were collected by reviewing patients' electronic medical records, including medical notes and the developmental assessments. Data on parents' ages, the existence of consanguineous marriage (a marriage between 2 individuals who are second cousins or closer), and problems during labor (such as mode of delivery) were obtained from the medical records. The study was ethically approved by the local ethical committee of KFSH-D which follow the ethical standards as was declared by Helsinki in 2020 review.

Children are diagnosed with GDD if they exhibit significant delays in 2 or more of the following developmental domains: daily living activities, motor skills, speech/language, cognitive abilities, and social/ emotional skills.

Search method. An electronic search was carried out in these databases: PubMed, The search terms (MeSH) used in these databases were ("epilepsy" OR "seizure") AND ("global developmental delay " OR "developmental delay "OR "learning disability")

Assessment tools. Participants were assessed for developmental delay using the Griffiths Scales of Mental Development for children aged zero to 2 years and for older children. These scales assess the five subscales of locomotor skills, personal and social skills, hearing and language/speech, eye and hand coordination, and performance. The Griffiths Scales are scored using reference standards that are obtained by averaging participants' scores on the five subscales. ${ }^{12,13}$

Statistical analysis. Data was entered and analyzed by using IBM statistical package for the social sciences 
Table 1 - Demographic parameters of study participants.

\begin{tabular}{lcccc}
\hline Parameters & \multicolumn{2}{c}{$\begin{array}{c}\text { Epilepsy } \\
\mathbf{n}=112\end{array}$} & \multicolumn{2}{c}{$\begin{array}{c}\text { Epilepsy free } \\
\mathbf{n}=88\end{array}$} \\
& $\mathbf{n}$ & $(\%)$ & $\mathbf{n}$ & $(\%)$ \\
\hline $\begin{array}{l}\text { Age } p=0.045 \\
\text { 3 months - 1 year }\end{array}$ & 7 & $(6.2)$ & 8 & $(9)$ \\
1-2 year & 16 & $(14.2)$ & 12 & $(13.6)$ \\
2-5 year & 23 & $(20.5)$ & 33 & $(37.5)$ \\
5-8 year & 33 & $(29.4)$ & 26 & $(29.5)$ \\
8-12 year & 33 & $(29.4)$ & 9 & $(10.2)$ \\
Sex $p=0.22$ & & & & \\
Males $(\mathrm{n}=113)$ & 59 & $(52.6)$ & 54 & $(61.3)$ \\
Females $(\mathrm{n}=87)$ & 53 & $(47.3)$ & 34 & $(38.6)$ \\
\hline
\end{tabular}

Table 2 - Pregnancy problems and epilepsy in GDD* patients.

\begin{tabular}{lcccc}
\hline Parameters & \multicolumn{4}{c}{ Pregnancy Problems } \\
& Yes & No & Total & $P$-value \\
\hline Epilepsy & 17 & 95 & 112 & $p=0.479$ \\
Epilepsy free & 15 & 73 & 88 & \\
Total & 32 & 168 & 200 \\
\hline \multicolumn{5}{c}{${ }^{*}$ Global developmental delay } \\
\hline
\end{tabular}

version for Windows, version 21 (IBM Corp., Armonk, N.Y., USA). Qualitative data were expressed as frequencies and percentage, such as sex and region. An Independent t-test was used to find the differences in the general characteristics between patients with epilepsy and patient without epilepsy. Throughout the study, a $p$-value less than 0.05 considered to be statistically significant.

Results. General characteristics of the participants. The current study included 200 pediatric patients diagnosed with GDD. Of the 200 participants, 113 were male and 87 were female (Table 1); their ages ranged from zero to 12 years. Participants were divided into 5 categories based on age: $7.5 \%(n=15)$ patients were between 3 months and one-year-old;14\% ( $\mathrm{n}=28)$ were between one and 2 years old; $28 \%(\mathrm{n}=56)$ patients were between 2 and 5 years old; $29.5 \%(n=59)$ patients were between 5 and 8 years old; and 21\% $(n=42)$ patients were between 8 and 12 years old (Table 1).

The participants were from different regions in the Eastern Province; $27.5 \%$ ( $n=55$, Figure 1) were from Dammam, $16.5 \% \quad(n=33)$ were from Qatif, $16.5 \%$ were from Al-Hasa, $16.5 \%$ were from other eastern rural areas, $10 \%(n=20)$ were from Hafar Al Batin, 8\%
Table 3 - Parental age and epilepsy in GDD patients:

\begin{tabular}{lcc}
\hline Parameters & Epilepsy & Epilepsy free \\
\hline Maternal age & & 42 \\
20-30 years & 44 & 36 \\
30-40 years & 42 & 7 \\
Above 40 years & 24 & 85 \\
Total & 110 & \\
P-value & $p=0.144$ & \\
Paternal age & & 6 \\
20-30 years & 5 & 18 \\
30-40 years & 40 & 72 \\
Above 40 years & 46 & \\
Above 40 years & 91 & \\
P-value & $p=0.003$ & \\
\hline
\end{tabular}

$(n=16)$ were from Khobar, and 5\% $(n=10)$ were from Jubail.

Regarding GDD subtypes, development delay was reported in $32.3 \%$ ( $n=63$ ) of the participants, learning disabilities were reported in $9.2 \%(\mathrm{n}=18)$, hyperactivity was reported in $12.8 \% \quad(n=25)$, speech delay was reported in $17.9 \%(\mathrm{n}=35)$, epilepsy-related syndromes were reported in $22 \%(n=43)$, and others issues were reported in $5.6 \%(\mathrm{n}=11)$.

Prevalence of epilepsy in participants with GDD. Of the participants with GDD, 56\% ( $\mathrm{n}=112)$ were diagnosed with epilepsy, and $44 \%(n=88)$ were epilepsy free. There was a significant age difference between GDD patients with epilepsy and those without epilepsy $(p=0.045$; mean difference $=0.43$; standard error $=0.16$; $95 \% \mathrm{CI}, 0.10,0.76)$. However, no significant differences in gender $(p=0.22)$ or region $(p=0.90)$ were found between the 2 groups (Table 1 ).

Of the participants with epilepsy, 36.1\% (n=39) had epilepsy-related syndromes, and 29.6\% ( $n=32)$ had developmental delay. Hyperactivity and speech delay were both reported in $11.11 \%(\mathrm{n}=12)$; learning disabilities and other issues were reported in $10.18 \%$ $(\mathrm{n}=11)$ and $1.8 \% \quad(\mathrm{n}=2)$ respectively. Levene's test revealed no significant differences between the epilepsy and non-epilepsy groups in subtypes of GDD $(p=0.273)$.

Prevalence of different types of seizures in GDD patients. Of the participants in the epilepsy group, $23.2 \%(n=26)$ had focal aware motor, $11.6 \%(n=13)$ had focal unaware motor, $4.4 \%(n=5)$ had focal unaware non-motor, $37.5 \%(\mathrm{n}=42)$ had generalized onset motor, $13.3 \%(n=15)$ had generalized onset non-motor, 9.8\% $(\mathrm{n}=11)$ had unknown onset motor, $4.4 \% \quad(\mathrm{n}=5)$ had 


\section{Number of patients per different regions in eastern province}

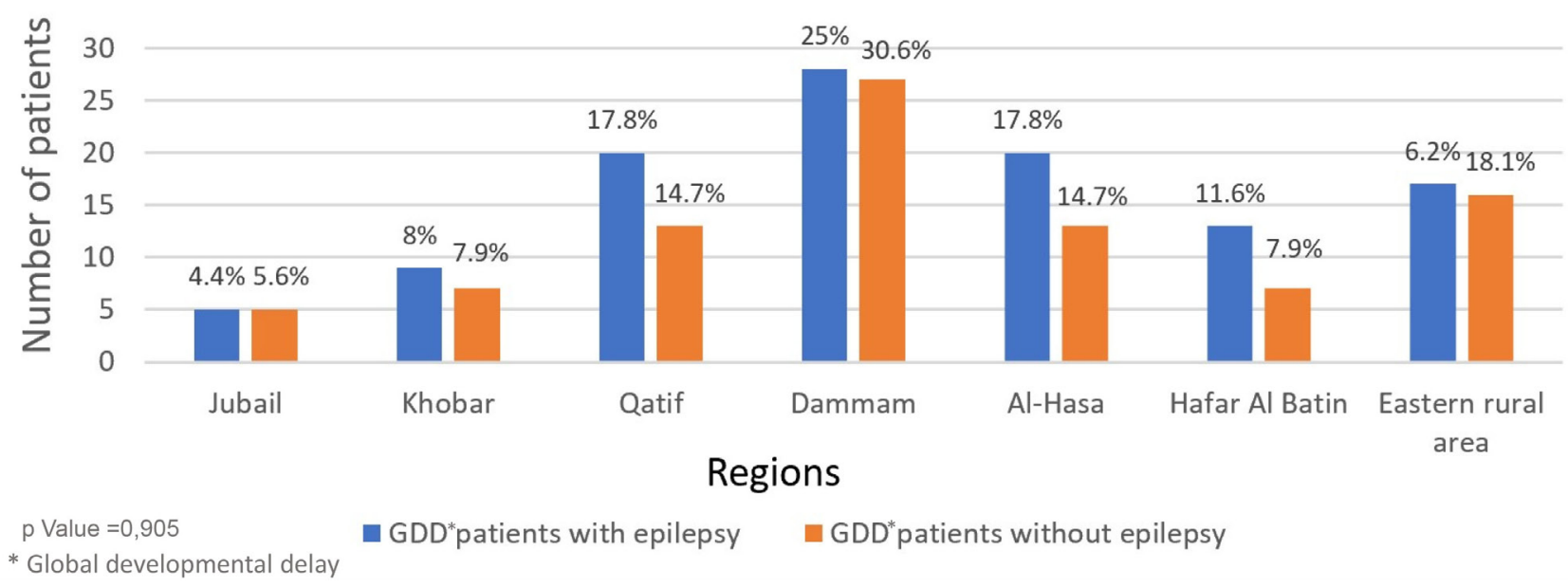

Figure 1 - Number of the recruited patients per region.

unknown onset non-motor and 11.6\% (n=13) had unclassified type.

Etiology classification of seizures in GDD patients. Of the participants in the epilepsy group, $57.1 \%(\mathrm{n}=64)$ had structural etiology, 25\% ( $n=28)$ had genetic, $6.2 \%$ $(\mathrm{n}=7)$ had infection, $19.6 \%(\mathrm{n}=22)$ had metabolic, and $11.6 \%(\mathrm{n}=13)$ had unknown type.

Pregnancy problems and seizures in GDD patients. Of the participants with epilepsy, problems during pregnancy occurred in $15 \%(n=17)$; there were no significant differences in problems during pregnancy between the epilepsy and non-epilepsy groups $(p=0.479$, Table 2).

Consanguineous marriage and seizures in GDD patients. Family consanguinity was found in $62.7 \%$ $(n=69)$ of the patients with seizure and $65.8 \%(n=56)$ in patient without seizure with no significant differences between the 2 groups ( $p=0.607$ )

Parental age and seizures in GDD patients. The mothers of $39 \%(n=44)$ of participants were 20 to 30 years old; the mothers of $37.5 \%(n=42)$ were 30 to 40 years old, and the mothers of $21 \%(n=24)$ were over 40. There were no significant differences in maternal age between the 2 groups $(p=0.144)$. The fathers of $41 \%(n=46)$ of participants were over 40 years old; the fathers of $35.7 \%(n=40)$ were 30 to 40 years old, and the fathers of $4.4 \%(n=5)$ were 20 to 30 years old. The paternal age of the epilepsy and non-epilepsy groups differed significantly $(p=0.003$; mean difference $=0.28$; standard error=0.09; 95\% CI, 0.10, 0.46, Table 3).
Discussion. The present study examines the prevalence of epilepsy in children with GDD, a topic that is currently under-studied. This was a hospitalbased retrospective study conducted at a tertiary care hospital in the eastern province of Saudi Arabia. As in previous similar studies, the sample was male dominated $(57.85 \%) \cdot{ }^{14,15}$ Of our participants, $28 \%$ were aged one to 2 years; $29.5 \%$ were aged 2 to 5 , and $21 \%(n=42)$ were aged 5 to 8 . Berg et $\mathrm{al}^{16}$ examined the frequency and risk factors of cognitive impairment in recently diagnosed epileptic children aged one month to 16 years. Age of onset was found to be a significant factor as $40.9 \%$ of participants who experienced epilepsy before 5 years old were cognitively impaired. ${ }^{16}$

In our study, $56 \%$ of GDD patients were diagnosed with epilepsy, and 44\% ( $n=88)$ were epilepsy free; there was a significant difference in age between GDD patients with and without epilepsy. A larger previous study compared the frequencies of medical, neurologic, developmental, and psychiatric conditions in children with epilepsy and in the general pediatric population. Nearly $80 \%$ of epileptic children has one or more comorbid disease. Also, the study showed that medical disorders were the highest comorbidity in children with epilepsy (55\%) followed by developmental/psychiatric disorders (43\%) and neurologic disorders (41\%). ${ }^{17}$ An etiological factor was identified in $65 \%$ of childhoodonset epileptic patients, most commonly geneticl presumed genetic (32\%) and structural $(29 \%){ }^{18}$ 
The most common type of seizure in our epilepsy group was generalized tonic seizures, which occurred in $44.6 \%$ of our participants; the second most common type was myoclonic seizures. This is similar to previous studies. ${ }^{14,15,19}$ Seizures with unknown onset were seen in $5.78 \%$ of our cases.

Although only $10.18 \%$ of our total participants showed evidence of learning disabilities, and no significant differences was found between the epilepsy and non-epilepsy groups, many previous studies suggested a relation between LD and epilepsy. Results of cohort study published in 2014 found that $76 \%$ of children with early onset epilepsy had learning disability. ${ }^{20}$ Fastenau et al, ${ }^{21}$ align with studies suggesting that the epileptic children are more prone to have LD than healthy ones. ${ }^{21}$

A 2014 prospective, community-based study of school-aged children with active epilepsy and behavioral disorders and/or cognitive impairments found that $40 \%$ had intellectual disabilities and 33\% had attention deficit/hyperactivity disorder. ${ }^{22}$ Another study examined behavioral problems and cognitive impairment in children with epilepsy aged 6 to 17 years and concluded that $34.6 \%$ of the participants have at least one behavioral problem. ${ }^{23}$ Psychosocial problems have also been observed in children with epilepsy but without severe non-verbal cognitive problems. ${ }^{6,7,20,21} \mathrm{On}$ the other hand, our results showed that approximately $11 \%$ of the total children had hyperactivity with no significant difference between epileptic and nonepileptic groups.

In our study, problems during pregnancy occurred in $15 \%$ of participants with epilepsy, with no significant differences between the epilepsy and non-epilepsy groups. Previous studies have observed a correlation between developmental delay and infants who experienced problems during pregnancy. ${ }^{24,25}$

Our finding around consanguineous marriage aligns with a previous prospective study in which children with recently diagnosed recurrent seizures were examined for clinical patterns of GDD; consanguinity was found in $29.7 \%$ of participants. ${ }^{26}$ In another previous study, interfamily marriages occurred in $57 \%$ of children with GDD. ${ }^{1}$

Study limitations. This a retrospective cross-sectional study, the convenience sample was taken from children under 12 who were treated in the pediatric neurology department of a hospital. Since admission bias may be a factor, our findings may not apply to the entire population in the eastern province of KSA.
Study implication. The present study is the first step in determining the risk factors contributing to high prevalence of epilepsy in GDD patients in eastern province in KSA, and further researches need to carry out to identify other risk factors to establish strategies to control them. Also, this study provides information for future researches which can guide the development of strategies for managing epilepsy in GDD patients in the eastern province, KSA.

Conclusion. Global developmental delay (GDD) occurs when children under 5 years old experience a significant delay in two or more developmental domains. This study examines the prevalence of epilepsy in GDD patients. It also examines the effects of other factors, including type of seizure, problems during pregnancy, consanguineous marriage, and advanced parental age. We find that the prevalence of epilepsy was high among children with GDD (56\%), and the most significant factors affecting the prevalence of epilepsy among these children were the type of seizure and advanced paternal age.

Acknowledgements. The authors gratefully would like to thank Scribendi Inc. Chatham, Ontario N7M ON3, Canada for English language editing.

\section{References}

1. Habibullah H, Albradie R, Bashir S. Identifying pattern in global developmental delay children: A retrospective study at King Fahad specialist hospital, Dammam (Saudi Arabia). Pediatr Rep 2019; 11: 8251.

2. Choo YY, Agarwal P, How CH, Yeleswarapu SP. Developmental delay: identification and management at primary care level. Singapore Med J 2019; 60: 119-123.

3. Alsharif MM, El-Fetoh NM, Ali GY, Alanazi KF, Alanazi AN, FalahAlanazi O, et al. Epilepsy as a health problem among school children in Turaif, Northern Saudi Arabia, 2017. Electron Physician 2017; 9: 5036-5042.

4. Kliegman R, Nelson W. Nelson textbook of pediatrics. 20th ed. Philadelphia (PA): Elsevier/Saunders; 2015.

5. Fisher RS, Cross JH, French JA, Higurashi N, Hirsch E, Jansen FE, et al. Operational classification of seizure types by the International League Against Epilepsy: Position Paper of the ILAE Commission for Classification and Terminology. Epilepsia 2017; 58: 522-530.

6. Fastenau PS, Johnson CS, Perkins SM, Byars AW, deGrauw TJ, Austin JK, et al. Neuropsychological status at seizure onset in children: risk factors for early cognitive deficits. Neurology 2009; 73: 526-534.

7. Bailet LL, Turk WR. The impact of childhood epilepsy on neurocognitive and behavioral performance: a prospective longitudinal study. Epilepsia 2000; 41: 426-431.

8. Bromley RL, Leeman BA, Baker GA, Meador KJ. Cognitive and neurodevelopmental effects of antiepileptic drugs. Epilepsy Behav 2011; 22: 9-16.

9. Nickels KC, Zaccariello MJ, Hamiwka LD, Wirrell EC. Cognitive and neurodevelopmental comorbidities in paediatric epilepsy. Nat Rev Neurol 2016; 12: 465-476. 
10. Vingerhoets G. Cognitive effects of seizures. Seizure 2006; 15: 221-226.

11. Rathouz PJ, Zhao Q, Jones JE, Jackson DC, Hsu DA, Stafstrom CE, et al. Cognitive development in children with new onset epilepsy. Dev Med Child Neurol 2014; 56: 635-641.

12. Griffiths R. The abilities of young children: A comprehensive system of mental measurement for the first eight years of life. Association for Research in Infant and Child Development; 1984.

13. Griffiths R. The Griffiths Mental Development Scales from Birth to 2 Years, Manual, the 1996 Revision. Henley: Association for Research in Infant and Child Development, Test agency; 1996.

14. Adhikari S, Sathian B, Koirala DP, Rao KS. Profile of children admitted with seizures in a tertiary care hospital of Western Nepal. BMC Pediatr 2013; 13: 43.

15. Mwipopo EE, Akhatar S, Fan P, Zhao D. Profile and clinical characterization of seizures in hospitalized children. Pan Afr Med J 2016; 24313.

16. Berg AT, Langfitt JT, Testa FM, Levy SR, DiMario F, Westerveld $\mathrm{M}$, et al. Global cognitive function in children with epilepsy: a community-based study. Epilepsia 2008; 49: 608-614.

17. Aaberg KM, Bakken IJ, Lossius MI, Lund Søraas C, Håberg SE, Stoltenberg C, et al. Comorbidity and Childhood Epilepsy: A Nationwide Registry Study. Pediatrics 2016; 138: e2016092120160921.

18. Sokka A, Olsen P, Kirjavainen J, Harju M, Keski-Nisula L, Räisänen $S$, et al. Etiology, syndrome diagnosis, and cognition in childhood-onset epilepsy: A population-based study. Epilepsia Open 2017; 2: 76-83.
19. Ahmed S, Alam ST, Rahman MM, Akhter S. Clinical profile of early childhood epilepsy: A cross sectional study in a tertiary care hospital. Mymensingh Med J 2016; 25: 96-101.

20. Sillanpää M. Learning disability: occurrence and long-term consequences in childhood-onset epilepsy. Epilepsy Behav. 2004; 5-: 937-944.

21. Fastenau PS, Jianzhao Shen, Dunn DW, Austin JK. Academic underachievement among children with epilepsy: proportion exceeding psychometric criteria for learning disability and associated risk factors. J Learn Disabil 2008; 41: 195-207.

22. Reilly C, Atkinson P, Das KB, Chin RF, Aylett SE, Burch V, et al. Neurobehavioral comorbidities in children with active epilepsy: a population-based study. Pediatrics 2014; 133: e1586-e1593.

23. Matonda-Ma-Nzuzi T, Mampunza Ma Miezi S, Mpembi MN, Mvumbi DM, Aloni MN, Malendakana F, et al. Factors associated with behavioral problems and cognitive impairment in children with epilepsy of Kinshasa, Democratic Republic of the Congo. Epilepsy Behav 2018; 78: 78-83.

24. Garfinkle J, Shevell MI. Cerebral palsy, developmental delay, and epilepsy after neonatal seizures. Pediatr Neurol 2011; 44: 88-96.

25. Nunes ML, Martins MP, Barea BM, Wainberg RC, Costa JC. Neurological outcome of newborns with neonatal seizures: a cohort study in a tertiary university hospital. Arq Neuropsiquiatr 2008; 66: 168-174.

26. Al-Sulaiman AA, Ismail HM. Clinical pattern of newlydiagnosed seizures in Saudi Arabia: a prospective study of 263 children. Childs Nerv Syst 1999; 15: 468-471.

\section{Statistics}

Excerpts from the Uniform Requirements for Manuscripts Submitted to Biomedical Journals updated November 2003. Available from www.icmje.org

Describe statistical methods with enough detail to enable a knowledgeable reader with access to the original data to verify the reported results. When possible, quantify findings and present them with appropriate indicators of measurement error or uncertainty (such as confidence intervals). Avoid relying solely on statistical hypothesis testing, such as the use of $P$ values, which fails to convey important information about effect size. References for the design of the study and statistical methods should be to standard works when possible (with pages stated). Define statistical terms, abbreviations, and most symbols. Specify the computer software used. 